\title{
Case Report: Intracranial Hypertension in Labour
}

\author{
Gunasena GGA ${ }^{\mathrm{a}}$, Godigamuwa $C^{\mathrm{b}}$, Bandara HGWAAK ${ }^{\mathrm{d}}$, Jayasundara DMCS ${ }^{\mathrm{e}}$, Hemapriya $\mathrm{S}^{\mathrm{f}}$
}

Idiopathic intracranial hypertension, Abducens nerve palsy, Induction of labour, Prostaglandins

\section{Abstract}

Neurological manifestations are seldom reported during labour with majority of cases being eclampsia. We report a case of 33-year-old lady in her second pregnancy developing features of raised intracranial pressure (bilateral Abducens nerve palsy and papilloedema) after administration of Prostaglandin E2 for induction of labour. Radiological imaging was unremarkable and the neurological symptoms spontaneously resolved within 24 hours of the delivery of the baby..

\section{INTRODUCTION}

Raised intracranial pressure (ICP) is rarely encountered in obstetric practice although it is common in neurology and neurosurgery. It can arise as a consequence of localized mass lesions (haematomas, neoplasms and abscesses), disturbance of CSF circulation (hydrocephalus), obstruction to major venous sinuses (cerebral venous thrombosis), diffuse brain oedema (encephalitis, meningitis) and idiopathic intracranial hypertension ${ }^{1}$.

Idiopathic intracranial hypertension (IIH) is a syndrome of elevated intracranial pressure with normal CSF composition and no evidence of hydrocephalus or mass lesion. IIH is most often seen in obese women of reproductive age $(19.3 / 100,000)$ and is reported only occasionally during pregnancy $^{2}$. It can occur in any trimester during pregnancy. It is postulated that

${ }^{a}$ Consultant Obstetrician and Gynaecologist, Consultant, District Base Hospital, Rikillagskada, Sri Lanka

${ }^{b}$ Consultant Physician, District Base Hospital, Rikillagaskada

'Registrar in Obstetrics and Gynaecology, Teaching Hospital, Kandy, Sri Lanka

${ }^{d}$ Senior Lecturer in Obstetrics and Gynaecology, Faculty of Medicine, University of Peradeniya, Sri Lanka

${ }^{e}$ Consultant Obstetrician and Gynaecologist, Teaching Hospital, Kandy, Sri Lanka

Correspondence: Dr. G. G. A. Gunasena MD (Obs \& Gyn), MRCOG (UK)

E-mail: asankagunasena@gmail.com

Competing interest: The authors report no conflict of interest thrombophilia and hypofibrinolysis seen in high estrogen conditions such as pregnancy, obesity, and polycystic ovarian syndrome lead to thrombosis of arachnoid villi and reduced CSF absorption, increasing the intracranial pressure ${ }^{2}$.

The most common symptoms are headache and visual disturbances including transient visual obscurations and diplopia. Treatment for IIH during pregnancy includes dietary control, carbonic anhydrase inhibitors

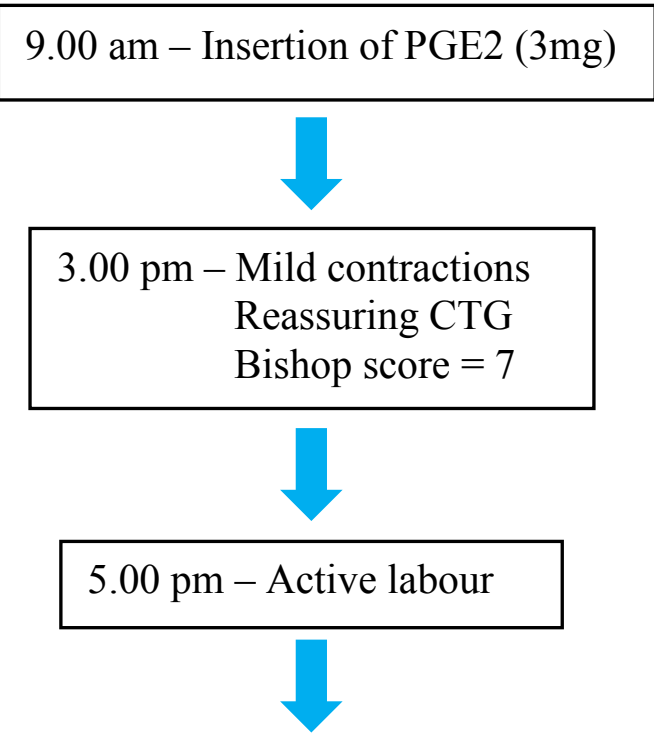

\section{$5.30 \mathrm{pm}$ - Bilateral Abducens nerve palsy (diplopia) Bilateral Papilloedema \\ Transferred to tertiary care center}

\section{$7.15 \mathrm{pm}$ - Caesarean section under GA} Indication: Pathological CTG

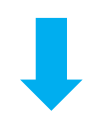

CT Brain - No hydrocephalus, venous sinus thrombosis or Space occupying lesion Spontaneous recovery within 24 hours

Time line of the case 
- acetazolamide (may be teratogenic), furosemide, corticosteroids and serial lumbar punctures ${ }^{2,3}$.

\section{CASE REPORT}

A 33-year-old mother of one child was admitted to ward at 40 weeks of gestation for confinement. Her BMI was 24.5 at the booking visit. She received shared care and did not have features suggestive of raised intracranial pressure such as headache or visual disturbance. A decision was made to induce labour at 40 weeks +2 days as she complained of persistently reduced fetal movements despite a normal biophysical profile and normal cardiotocographs (CTGs). The Bishop score was 5 and vaginal Prostaglandin E2 $(3 \mathrm{mg})$ was administered for induction of labour around $9.00 \mathrm{am}$ in the morning.

Around $4.00 \mathrm{pm}$, the uterine contractions became more intense and she was sent to the labour ward at $5.00 \mathrm{pm}$ in active labour. She received Entonox for analgesia and fetus was monitored with continuous CTG. Around $5.30 \mathrm{pm}$, she complained of a headache and shortly afterwards she became acutely distressed with sudden onset blurred vision due to diplopia. Her blood pressure was $130 / 70 \mathrm{mmHg}$ and vaginal examination revealed a $4 \mathrm{~cm}$ dilated cervix with intact membranes. The CTG showed uterine hypertonus with occasional variable decelerations. She was examined by the consultant physician and bilateral Abducens nerve (VI nerve) palsy and bilateral papilledema were detected. The clinical diagnosis of intracranial hypertension was made and she was transferred to the nearest tertiary care center for further management. On arrival at the tertiary care center, she was haemodynamically stable; but the CTG was pathological with uterine hyperstimulation. The delivery needed to be expedited and an emergency caesarean section was planned. Without radiological imaging to exclude a space-occupying lesion prior to surgery, the anaesthetic team decided provide general anaesthesia. She had an uncomplicated caesarean section around 7.15 p.m. and the baby was healthy at birth. After the delivery, she had a non-contrast CT scan which was unremarkable (no hydrocephalus, venous sinus thrombosis or space occupying lesion). She was admitted to the intensive care unit for observation and no special medication was needed as her neurological manifestations spontaneously resolved within 24 hours of the delivery of the baby. All other blood and urine investigations were normal. She was discharged from medical care on day five.

\section{DISCUSSION}

In this case scenario, intracranial hypertension was clinically diagnosed by the presence of bilateral Abducens nerve palsy and papilledema. Considering the acute nature of the clinical manifestations, the possibility of a vascular lesion such as venous sinus thrombosis or intracranial haemorrhage needed to be excluded as a priority. But her radiological imaging did not reveal any intracranial pathology leaving a diagnostic dilemma.

Idiopathic intracranial hypertension (IIH) was considered a probable diagnosis in this patient. The diagnosis of IIH is by exclusion using modified Dandy criteria ${ }^{4}$.

\begin{tabular}{|l|l|}
\hline \multicolumn{2}{|c|}{ Modified Dandy Criteria } \\
\hline 1. & $\begin{array}{l}\text { Signs and/or symptoms of } \\
\text { increased intracranial pressure. }\end{array}$ \\
\hline 2. & $\begin{array}{l}\text { Documented elevated } \\
\text { intracranial pressure }(>250 \mathrm{~mm} \\
\text { water). }\end{array}$ \\
\hline 3. & Normal CSF composition. \\
\hline 4. & $\begin{array}{l}\text { No evidence of hydrocephalus, } \\
\text { mass, structural, or vascular } \\
\text { lesion on imaging. }\end{array}$ \\
\hline 5. & $\begin{array}{l}\text { No localizing neurological signs } \\
\text { except a unilateral/ bilateral VI } \\
\text { nerve paresis. }\end{array}$ \\
\hline
\end{tabular}

\section{Table1. Modified Dandy Criteria}

Although this patient had signs of increased intracranial pressure like papilledema and bilateral VI nerve palsy, neither intracranial pressure nor CSF composition was measured as she had general anaesthesia for caesarean section instead of spinal anaesthesia. A lumbar puncture would have been a better option as it is one of the treatment modalities in IIH allowing CSF drainage and reduction in CSF pressure ${ }^{2}$. However general anesthesia had to be considered in the absence of prior radiological imaging.

With the assumption of asymptomatic IIH in this patient, an acute and a transient increase in the intracranial pressure can be expected if she had adopted Valsalva maneuver in response to uterine hypertonus secondary to PGE2 administration. But in the absence of confirmatory evidence, IIH still remains a clinical diagnosis.

An idiosyncratic reaction to Prostaglandins was also considered a possibility. Although mild visual disturbances are reported with PGE2, there are no case reports of bilateral VI nerve palsy after PGE2 administration. Contrarily, PGE2 induces vascular relaxation and animal studies have shown that PGE2 decreases intracerebral pressure ${ }^{5}$.

All her neurological symptoms and signs improved with the gradual reduction of intracranial pressure after the delivery of the baby. The final diagnosis is still open for discussion.

\section{REFERENCES}

1. Dunn LT. Raised Intracranial Pressure. $J$ Neurol Neurosurg Psychiatry. 2002;73(suppl I):i23-i27

2. Badve M, McConnell MJ, Tanmay $\mathrm{S}$, et.al. Idiopathic Intracranial Hypertension in Pregnancy Treated with Serial Lumbar Punctures. International Journal of Clinical Medicine. 2011; 2:9-12.

3. Chih A, Patel B. Idiopathic Intracranial Hypertension in Pregnancy. Fed Pract. 2015 November;32(11):36-39

4. Friedman DI, Jacobson DM. Diagnostic criteria for idiopathic intracranial hypertension. Neurology. 2002;59(10):1492-5.

5. Nakano J, Chang AC, Fisher RG. Effects of prostaglandins E1, E2, A1, $\mathrm{A} 2$, and $\mathrm{F} 2$ on canine carotid arterial blood flow, cerebrospinal fluid pressure, and intraocular pressure. J Neurosurg. 1973; 38 (1): 32-39. 